\title{
EXTENDED OPTIMA AND EQUILIBRIA FOR CONTINUOUS GAMES. I. GENERAL RESULTS
}

\author{
D. J. GATES AND M. WESTCOTT
}

(Received 21 May 1980)

(Revised 9 October 1980)

\begin{abstract}
In this, the first of three papers, we examine conditions, derived previously, which specify the equilibrium solutions of an adjustment process for $N$ players engaged in a game with continuous (in fact, continuously differentiable) payoff functions, where each player's strategy is to choose a single real number. It is equivalent to the basic form of quantity-variation competition between $N$ firms. The conditions are related to a new optimum which takes account of the ability of firms, or coalitions of firms, to discipline another firm that tries to increase its own profit. Closely related optima are also introduced and analysed. The new optima occupy $N$-dimensional regions in the strategy space, and contain the optima of Cournot, Pareto, von-Neumann and Morgenstern, and Nash as special cases.
\end{abstract}

\section{Introduction}

An adjustment process for many firms in competition, with no knowledge of the payoff structure of the market, was studied by Gates, Rickard and Wilson [3] (see also [2], [8], [4], [5] and [11]). It was found numerically, for the case of 2 firms, that the adjustment process converges for all sensible initial conditions. In general, with $N$ firms, all equilibrium states of this process were proved to satisfy the new condition: there exists a non-zero, positive-semi-definite (psd) matrix $\boldsymbol{A}_{i j}$ $(i, j=1,2, \ldots, N)$ such that

$$
\sum_{j=1}^{N} A_{i j} J_{i j}=0 \quad \text { for } i=1,2, \ldots, N,
$$

where $J_{l}(\sigma)$ is the payoff (or profit) to firm $i$ when firms 1 to $N$ produce 
quantities $\left(\sigma_{1}, \ldots, \sigma_{N}\right)=\sigma$ respectively of the competing product (during one business period) and

$$
J_{i j}(\boldsymbol{\sigma})=\partial J_{i} / \partial \sigma_{j}
$$

Thus, (1.1) restricts the set $E$ of equilibrium outputs $\sigma$ to a set $M$ say; that is, $E \subseteq M$.

Also, in [3], the duopoly $(N=2)$ case of $(1.1)$ was shown to be equivalent to the new condition

$$
J_{11} J_{22} \operatorname{det}\left(J_{i j}\right) \leqslant 0 \text {, }
$$

involving only the $J_{i j}$ 's. The resulting set of $\sigma$ 's appeared to coincide with the set of solutions to which the adjustment process converged numerically, suggesting that (1.1) not only restricts, but completely defines, the equilibrium states in the duopoly case; that is, $E=M$.

We seek an interpretation of (1.3) in terms of the game with payoffs $J_{1}$ and $J_{2}$. The condition

$$
J_{i i}=0
$$

is just that which maximizes the profit of firm $i$ on the assumption that the other firm holds its output fixed. Thus the well known Cournot optimum, for which

$$
J_{11}=J_{22}=0 \text {, }
$$

results when both firms adopt this policy. The condition

$$
\operatorname{det}\left(J_{i j}\right)=0
$$

includes the Pareto optimal solutions of the game and the threat curve of Mayberry, Nash and Shubik [9]. By definition, $\sigma$ is Pareto optimal if any change in $\sigma_{1}$ or $\sigma_{2}$ results in a decrease in $J_{1}$ or $J_{2}$ or both. Since no firm wants a reduction in profit, a Pareto optimum is usually regarded as a fair or cooperative solution to the bargaining problem which rational, fully informed (as to $J_{1}$ and $J_{2}$ ) firms might agree to adopt.

We note that (1.4), (1.5) and (1.6) are all special cases of (1.3), which suggests that (1.3) may itself have an interpretation as an optimal solution of the game. We show that this is indeed so.

We further show that, for all $N$, the matrix equations (1.1) can be related to a collection of conditions like (1.3) on the $J_{i j}$ 's; in particular, a point $\sigma$ satisfying these conditions is always in $M$. The conditions define a set $O_{1}^{W}$ of $\sigma$ which are optima of a new type. The boundary points of $O_{1}^{W}$ are given by the equations

$$
\Delta_{S}=0 \text { for } S \subseteq(1, \ldots, N) \text {, }
$$

where the $\Delta_{S}$ are principal minor determinants of the matrix $J_{i j}$. They are therefore related to Pareto optimal, or threat, solutions of games between subsets of the firms. In the following paper we prove that, for an important class of $J_{i}$ 's, $O_{\mathrm{I}}^{W}$ and $M$ coincide. 
These results support the widely held belief (for example, [1]) that firms acting independently with no knowledge of the profit functions of the market, will evolve towards an optimal or cooperative solution of the underlying game. Since much of economic theory is based on beliefs of this sort, mathematical proofs of their validity are desirable, and this is a motivation for the present work.

Besides the optima $O_{1}^{W}$, we introduce related optima which are more restrictive for $N>2$, but less restrictive than the Pareto optimum. All our new optima are based upon observing the consequences which any firm faces if it makes an adjustment (a small change in its $\sigma_{i}$ ) which increases its own profit. If some other firms can subsequently conspire to make adjustments which restore their own original profits while reducing the profit of the given firm below its original value, then the given firm would be disinclined to make the first adjustment. We call such a firm disciplinable. When all firms are disciplinable they all will tend not to make adjustments, and so we have a type of optimum.

By contrast the Pareto optimum involves only the immediate losses of firms when they make adjustments. It makes no allowances for responses to adjustments, a process which is obviously important in real markets. The Pareto optima comprise a relatively restricted set, being only an $(N-1)$ dimensional manifold in the $N$ dimensional set of $\sigma$ 's. The even more restrictive game-theoretic optima such as the Cournot optimum, the cooperative optima of VonNeumann and Morgenstern [10], and the non-cooperative optima of Nash (see [9]) with and without side payments, comprise even smaller sets in $\sigma$-space, sometimes a unique point, or they may not even exist for pure strategies. They also assume considerable knowledge on the part of the firms, both of the payoff functions and of the mathematical complexities of game theory.

For these reasons, the opinion is often expressed that these restrictive optima have limited application to economic modelling. Rather, they describe theoretical possibilities open to sophisticated bargainers. To model real economies one requires much broader optima that include all sorts of bargaining behaviour, even of the most naive and ill-informed competitors.

Our new optima come closer to meeting this requirement. They form much larger sets, $N$-dimensional in fact, and contain all the previous optima including the Pareto optima. They do not imply nearly as much knowledge or mathematical expertise on the part of the firms, although they do not exclude it. Firms can, in a sense, learn that they are in an optimum state of our type through being disciplined.

The present paper is devoted to general results relating the new optima to the adjustment equilibria $E$ and to the standard optima for general continuous games. Only pure strategies are considered.

In the second paper [6] of this series we consider a narrower class of functions $J_{i}$ with special relevance to competing firms. Much stronger results can then be 
obtained, and the optima can be illustrated. We also study the adjustment process of [3] for 3 firms numerically, and find that all of the equilibrium solutions defined by (1.1) appear to be generated. This indicates, as in the 2-firm case, that (1.1) not only restricts but completely defines the equilibrium states of the adjustment process.

In the third paper [7] it is shown that the new optima provide an improved explanation of some bargaining experiments.

\section{The extended optima}

We begin by defining the concept disciplinable more precisely together with the new optima based upon it. We suppose throughout that each of the $N$ firms, $i=1,2, \cdots N$, has payoff or profit $J_{1}(\sigma)$ which is a continuously differentiable function of each of the outputs $\sigma_{1}, \ldots, \sigma_{N}$ of the firms, and that $J_{i}(\sigma)$ is not constant on any interval of $\sigma_{i}$ values. We normally think of the profits and outputs as applying to one business period (a month perhaps). Firm $i$ has direct control only over its own output $\sigma_{t}$.

DeFINITION 1. The market is said to be in state $\sigma$ when the outputs are given by $\boldsymbol{\sigma}$.

Definition 2. $A$ coalition is a subset of the set $(1,2, \ldots, N)$ of firms.

Definition 3. In state $\sigma$, firm $i$ is said to be disciplinable by a coalition $C$ from the other firms if

$$
J_{i}\left(\boldsymbol{\sigma}+\varepsilon \mathbf{e}_{i}\right)<J_{i}(\boldsymbol{\sigma})
$$

for every sufficiently small $\varepsilon \neq 0$, or if, for every sufficiently small $\varepsilon$ such that

$$
J_{i}\left(\boldsymbol{\sigma}+\varepsilon \mathbf{e}_{i}\right)>J_{i}(\boldsymbol{\sigma})
$$

there are $\delta_{j}$ for all $j \in C$ such that

$$
\begin{gathered}
J_{i}\left(\boldsymbol{\sigma}+\varepsilon \mathbf{e}_{t}+\sum_{j \in C} \delta_{j} \mathbf{e}_{j}\right)<J_{\imath}(\boldsymbol{\sigma}), \\
J_{k}\left(\boldsymbol{\sigma}+\varepsilon \mathbf{e}_{t}+\sum_{j \in C} \delta_{j} \mathbf{e}_{j}\right)=J_{j}(\boldsymbol{\sigma}) \quad \text { for all } k \in C,
\end{gathered}
$$

and $\delta_{j} \rightarrow 0$ as $\varepsilon \rightarrow 0$, for all $j \in C$, where $\mathbf{e}_{j}$ is a vector with 1 in the $j$ th place and zeros elsewhere.

In other words, a small adjustment by firm $i$ either results directly in a reduction in profit or can be countered by some coalition from the other firms 
whose members can restore their original profits and leave firm $i$ with a net reduction in profit. We denote the set of such states $\sigma$ by $D_{i, C}$.

A variety of optima can be based on the concept of discipline.

Definition 4. A state $\sigma$ is a type I optimum if at least one firm can be disciplined by a coalition from the other firms.

Definition 5. A state $\sigma$ is a type II optimum if at least one firm can be disciplined by the coalition of all the other firms.

Definition 6. A state $\sigma$ is a type III optimum if every firm can be disciplined by a coalition from the other firms.

DEFINITION 7. A state $\sigma$ is a type IV optimum if every firm can be disciplined by the coalition of all of the other firms.

To say that a coalition of all, rather than a subset, of the other firms is involved is a greater restriction, because it requires that all of the other firms must be able to restore their original profits.

Our notion of firms forming a defensive coalition seems to be new in game theory. Previously, coalitions which give a benefit to the partners have been widely studied following the basic work of von Neumann and Morgenstern [10].

One can see that idealized firms in a bargaining situation and knowing the $J_{t}$ 's (that is, playing a game with complete information) would find an optimum state of type III or type IV acceptable. This is especially true if firms can make only small changes in their outputs (as is usual in practice). Every firm would be reluctant to depart from such a state because each would risk a reduction in short-term profit.

The type IV optimum is the most acceptable to all firms, because here every firm is not only reluctant to move through risk of being disciplined, but also belongs to every disciplining coalition and is consequently always protected.

Similar interpretations can be given for the other optima, types I and II. One imagines that the firms know only that at least one firm can be disciplined, but not which firm this is. Each firm therefore settles for this optimum in order to avoid the risk that it might itself be the susceptible firm.

These optima have an important relation to the original adjustment process [3] as will be seen in the following section (Theorems 5 and 6), and in [6].

We look for conditions for disciplinability which are independent of $\varepsilon$ and $\delta_{j}$ 's. We denote the set of all firms $(1,2, \ldots, N)$ by $\Lambda$, and the determinant of the submatrix $J_{i j}, i, j \in S$, for a subset $S \subseteq \Lambda$ of the firms, by $\Delta_{s}$. Thus $\Delta_{i}=J_{i i}$, and $\Delta_{\Lambda}$ is the determinant of the whole $J_{i j}$ matrix. 
Theorem 1. (a) If $\boldsymbol{\sigma} \in D_{i, C}$ then $\Delta_{i} \Delta_{C} \Delta_{C u i} \leqslant 0$.

(b) If $\Delta_{i} \Delta_{C} \Delta_{C u i}<0$ then $\sigma \in D_{i, C}$.

(A proof is given in Section 4).

Ideally one would like a one-to-one characterization of $D_{i, C}$ in terms of the $\Delta$ 's, but such a characterization would be extremely cumbersome in the ensuing applications (one would need to keep account continually of the vanishing of higher order derivatives of the $J_{i}$ 's). The set where $\Delta_{t} \Delta_{C} \Delta_{C \cup i}=0$ which is not wholly identified with $D_{i, C}$ has, in any case, zero $N$-dimensional volume. To simplify the presentation we introduce weak and strong versions of $D_{i, C}$.

Definition 8. Player $i$ is said to be weakly disciplinable by $C$ if $\Delta_{i} \Delta_{C} \Delta_{C U i}<0$.

Definition 9. Player $i$ is said to be strongly disciplinable by $C$ if $\Delta_{t} \Delta_{C} \Delta_{C \cup i}$ $<0$.

The corresponding sets of states $\sigma$ are denoted by $D_{i, C}{ }^{W}$ and $D_{i, C}{ }^{s}$, so that

$$
D_{i, C} s \subseteq D_{i, C} \subseteq D_{i, C}{ }^{w} \text {. }
$$

In typical economic applications one finds that $D_{i, C}{ }^{W}$ comprises simply $D_{i, C}$ and its boundary points, making weakly disciplinable an appealing concept. Similarly, $D_{i, C} S$ usually comprises $D_{i, C}$ without its boundary points. Unfortunately this does not hold in general: for example, if

$$
J_{1}=\left(\sigma_{1}-\sigma_{2}\right)\left(1-\sigma_{2}\right)+1
$$

and $J_{2}=\left(\sigma_{2}-\sigma_{1}\right)\left(1-\sigma_{1}\right)+1$, one obtains

$$
\Delta_{1} \Delta_{2} \Delta_{1,2}=2\left(1-\sigma_{1}\right)\left(1-\sigma_{2}\right)\left(\sigma_{1}-\sigma_{2}\right)^{2} \text {, }
$$

so that $\sigma_{1}=\sigma_{2}$ belongs to $D_{1,2}{ }^{W}$ but not to the boundary of $D_{1,2} s$.

On the other hand, $D_{i, C} s$ is equivalent to a set of linear constraints.

THEOREM 2. The state $\sigma \in D_{i, c} s$ if and only if for any $\alpha_{i}$ such that

$$
J_{i i} \alpha_{i}>0
$$

there exist unique $\alpha_{j}(j \in C)$ such that

$$
\sum_{j \in C \cup i} J_{i j} \alpha_{j}<0
$$

and

$$
\sum_{j \in C \cup i} J_{k j} \alpha_{j}=0 \text { for all } k \in C
$$

(A proof is given in Section 4). 
This theorem is used in the proof of Theorem 5.

Weak and strong optima of types I to IV are defined like their unqualified counterparts. The corresponding sets $O_{\mathrm{I}}^{W}$ to $O_{\mathrm{IV}}^{W}$ and $O_{\mathrm{I}}^{S}$ to $O_{\mathrm{IV}}^{S}$ clearly satisfy

$$
O_{k}^{S} \subseteq O_{k} \subseteq O_{k}^{W}, \quad k=\text { I, II, III, IV. }
$$

\section{Results comparing optima and equilibria}

It follows immediately from the preceding definitions that the weak optima of types I to IV are equivalent to the following conditions respectively:

TYPE I. $\Delta_{i} \Delta_{C} \Delta_{C \cup i} \leqslant 0$ for at least one $i \in \Lambda$ and at least one $C \subseteq \Lambda-i$.

TYPE II. $\Delta_{i} \Delta_{\Lambda-i} \Delta_{\Lambda} \leqslant 0$ for at least one $i \in \Lambda$.

TYPE III. $\Delta_{i} \Delta_{C} \Delta_{C u i} \leqslant 0$ for every $i \in \Lambda$, and at least one $C \subseteq \Lambda-i$ for every $i$.

TYPE IV. $\Delta_{t} \Delta_{\Lambda-i} \Delta_{\Lambda} \leqslant 0$ for every $i \in \Lambda$.

For the strong optima one just replaces $\leqslant$ everywhere by $<$.

It is now straightforward to relate the weak optima to standard optima.

Theorem 3. The Pareto and Cournot optima are weak optima of all of the types I to IV.

Proof. By the definition of a Pareto optimal state $\sigma$ there is no sufficiently small $\delta$ such that $J_{1}(\sigma+\delta)>J_{i}(\sigma)$ for all $i$, and hence no solution of the equations $\Sigma_{j} J_{i j} \delta_{j}=\nu_{j}$ if every $\nu_{j}>0$. Thus $\sigma$ satisfies

$$
\Delta_{\Lambda}=0 \text {, }
$$

which in turn satisfies (3.1) to (3.4). The Cournot optimum satisfies

$$
\Delta_{i}=0 \text { for all } i \in \Lambda \text {, }
$$

which also satisfies (3.1) to (3.4).

In the case of 2 firms, (3.5) yields both a threat curve and a Pareto optimal curve [9]. These in turn contain a variety of optima or solutions: the von Neumann and Morgenstern solution, the cooperative solution with side-payments, the cooperative solution without side-payments, and the threat solutions of Nash with and without side-payments. Consequently, all of these solutions are special cases of any of our weak optima, as discussed in Section 1. We are referring as always to pure strategies.

It is clear also from the above that no Cournot or Pareto optimal state belongs to any of the strong optima $O_{\mathrm{I}}^{S}$ to $O_{\mathrm{IV}}^{S}$. However, they are usually boundary points of these strong optima. 
The connection between our basic (unqualified) optima, $O_{1}$ to $O_{\mathrm{IV}}$, and the traditional optima is somewhat deeper. Let $P O^{\prime}$ denote the set of $\sigma$ 's which are Pareto optimal and for which rank $J_{i j}=N-1$.

Theorem 4. The Cournot and $P O^{\prime}$ optima belong to $O_{\mathrm{IV}}$ and hence to $O_{\mathrm{I}}, O_{\mathrm{II}}$ and $O_{\mathrm{III}}$

(A proof is given in Section 5).

The points $\sigma$ which are Pareto optimal but not in $P O^{\prime}$ have relatively zero measure, since they belong to the sets where $\Delta_{\Lambda-i}=0$ for at least one $i$, which have an $(N-2)$-dimensional intersection with the set where $\Delta_{\Lambda}=0$. For example, they comprise only points in the 2-firm case.

We now pursue the relation between the optima and the matrix condition (1.1). For example, one can easily see that a Cournot optimum satisfies (1.1) with

$$
A_{i j}= \begin{cases}1 & \text { if } i=j \\ 0 & \text { if not }\end{cases}
$$

and a Pareto optimum satisfies (1.1) with

$$
A_{i j}=x_{i} x_{j}
$$

and suitable $x_{i}^{\prime}$ 's. These are special cases of the following result.

THeOREM 5. A state $\sigma \in O_{1}^{W}$, and consequently any other of our optimal states, satisfies the matrix condition (1.1).

(A proof is given in Section 6).

For 2 firms one can say much more.

THEOREM 6. For $N=2$, weak optimality of types I to IV and the matrix condition (1.1) are all equivalent, that is $O_{\mathrm{I}}^{W}=O_{\mathrm{II}}^{W}=O_{\mathrm{III}}^{W}=O_{\mathrm{IV}}^{W}=M$.

Proof. By (3.1) to (3.4) with $N=2$, all optima $O_{\mathrm{I}}^{W}$ to $O_{\mathrm{IV}}^{W}$ reduce to

$$
\Delta_{1} \Delta_{2} \Delta_{12} \leqslant 0 \text {. }
$$

But the equivalence of (3.9) and (1.1) was proved in Theorem 2 of [3].

An alternative proof of Theorem 6 is given in Section 6 . It shows more closely the relation between production increments and the $A_{i j}$ 's of (1.1).

As mentioned at the beginning of Section 1, the equilibrium states $E$ satisfy the matrix condition (1.1), that is $E \subseteq M$. Theorem 6 now implies that $E \subseteq O_{k}^{W}$ 
( $k=\mathrm{I}, \ldots, \mathrm{IV})$ in the 2 -firm case. The numerical evidence that $E=M$ implies that $E=O_{k}^{W}$, so that every equilibrium state would be a weak optimum. In the long-term, myopic adjustment process of [3] yielding $E$, each firm's sole action is to maximize a crude estimate of its profit function. Mathematically it is most surprising that this process leads to optima that seem to imply greater knowledge and a bargaining between the firms.

On the other hand it supports the kind of belief, which is implicit in much of economic theory [1], that firms acting independently with little knowledge of the market structure (the $J_{i}$ 's) can arrive at an optimum solution of the underlying market game.

In the 2-firm case one can understand in a superficial way why the adjustment process of [3] leads to the type I optimum. The adjustment yrocess presumably has the property that, near such an optimum, it dictates a suitable disciplinary move to a firm when a profit-increasing move is dictated to the other. (We say "dictates", because it is not a conscious intention). Consequently it will not admit any profit changing moves, and hence gives an equilibrium. To understand this properly one would need a deeper analysis of the adjustment process of [3] but we have not succeeded in this.

For 3 or more firms we have only that $O_{k}^{W} \subseteq M$ (Theorem 5) and $E \subseteq M$, which does not necessarily imply any overlap of $O_{k}^{W}$ and $E$. However, a reverse implication giving $O_{1}^{W}=M$ is proved in Theorem 1 of [6] for an important class of $J_{i}$ 's. Thus $E \subseteq O_{\mathrm{I}}^{W}$ in this case, so that equilibria are again optima.

Since $O_{\mathrm{I}}^{W} \subseteq M$ and $E \subseteq M$, in general, the set $\sim M$ of $\sigma$ 's not in $M$ is a subset of $\sim O_{1}^{W}$ and $\sim E$. Thus a knowledge of $\sim M$ is useful in finding $\sigma$ 's excluded from $O_{1}^{W}$ and $E$. For general $J_{i}$ 's and $N>3$, we have only limited results about $\sim M$.

Theorem 7. Suppose that $J_{i j}$ 's satisfy (1.1). Then for any $H_{1}, \ldots, H_{N}$, the leading principal minor determinants $\Delta^{(1)}, \ldots, \Delta^{(N)}$ of the matrix $H_{i} J_{i j}+H_{j} J_{j i}$ are neither all positive nor satisfy

$$
(-1)^{i} \Delta^{(i)}>0 \text { for all } i \text {. }
$$

(A proof is given in Section 7).

Making various choices for the $H_{i}$ 's leads to various disallowed regions, as illustrated after Theorem 8.

We define

$$
E_{i j k}=\left\{\frac{1}{2}(N-2)\left(J_{j k} X+J_{k j} X^{-1}\right)\right\}^{2}
$$

where

$$
X=\left\{J_{i} J_{k i} /\left(J_{j i} J_{i k}\right)\right\}^{1 / 2}
$$


TheOrem 8. Assume that the $J_{i j}$ 's satisfy (1.1).

(a) Suppose also that $J_{i j} \leqslant 0$ for all $i \neq j$. Then the region

$$
B_{0}=\left\{\Delta_{S}>0 \text { for all } S \subseteq \Lambda\right\}
$$

is excluded.

(b) Suppose also that $J_{i j}<0$ for all $i \neq j$. Then the regions

$$
\begin{aligned}
B_{i}=\{ & J_{i i}<0, J_{j j}>0 \text { for all } j \in \Lambda-i, \\
& \left.J_{j j} J_{k k}>E_{i j k} \text { for all }(j, k) \subseteq \Lambda-i, j \neq k\right\}
\end{aligned}
$$

are excluded.

(A proof is given in Section 8).

The condition $J_{\imath j} \leqslant 0$ simply states that firm $i$ 's profit does not increase if firm $j$ alone makes a small increase in its output $\sigma_{j}$. One would expect this to apply usually.

Part (b) can be extended to include $J_{i j}=0$, but various cases need to be considered, and we shall not elaborate on these.

If $J_{i j}=K_{i} L_{j}$ for $i \neq j$ and some $K_{i}$ and $L_{j}$, and if $N=3$ then $E_{i j k}=J_{j k} J_{k j}$ and the condition $J_{j} J_{k k} \geqslant E_{i j k}$ reduces to $\Delta_{j k} \geqslant 0$. We see therefore that the determinants $\Delta_{S}$ play a role in excluding $\sigma$ 's as solutions of (1.1). This form of $J$, arises naturally in economics, as discussed in [6]. We find (see Theorem 4 of [6]) that Theorem 8 gives all the excluded $\sigma$ 's for $N=3$ and such $J_{i}$ 's.

For $N>3$ and such $J_{1}$ 's, Theorem 8 is improved by Theorem 4 of [6]. For example, let $J_{i}=\sigma_{i}\left(1-\sigma_{T}\right), i=1, \ldots, 4$, where $\sigma_{T}=\Sigma_{i} \sigma_{i}$, and $y_{i}=$ $\sigma_{i} /\left(1-\sigma_{r}\right)$. Suppose $y_{1}>1, y_{2}<1, y_{3}<1$ and $y_{4}<1$. Then the condition $J_{j j} J_{k k}>4 J_{j k} J_{k j}$ is satisfied for $(j, k)=(2,4)$ and $(3,4)$ if $y_{4}$ is sufficiently small (that is, if $\sigma_{4}$ is sufficiently small), and is satisfied for $(j, k)=(2,3)$ if

$$
y_{2}+y_{3}<1-3 y_{2} y_{3} \text {. }
$$

But Theorem 4 of [6] excludes the larger region $y_{2}+y_{3}<1$.

Theorem 7 of the present paper is not easy to apply because it requires a judicious choice of the $H_{i}$ 's. We shall not examine its general consequences here. For the above example it gives a stronger result than Theorem 8 . One chooses $y_{2}=y_{3}=\alpha, H_{1}=\alpha, H_{2}=H_{3}=-y_{1}$ and $H_{1}=-h y_{1} / 2$ for $h>0$. One then eventually finds that all of $y_{2}=y_{3}<\frac{1}{2}$ is excluded, in agreement with Theorem 4 of [6]. From (3.15), Theorem 8 excludes only the subset $y_{2}=y_{3}<\frac{1}{3}$ of $y_{2}=y_{3}$.

\section{Proofs of Theorems 1 and 2}

From the definition of $D_{i, C}$ there are two possibilities. The first is that (2.1) holds, so that $\Delta_{i}=0$, in agreement with part (a) of Theorem 1. Alternatively, 
(2.2), (2.3) and (2.4) hold, which implies that for any $\alpha_{i} \neq 0$ such that

$$
J_{i i} \alpha_{t} \geqslant 0 \text {, }
$$

we can find $\alpha_{j} \neq 0(j \in C)$ such that

$$
\sum_{J \in C \cup \imath} J_{i j} \alpha_{j} \leqslant 0
$$

and

$$
\sum_{j \in C \cup i} J_{k j} \alpha_{j}=0 \text { for all } k \in C .
$$

If $\Delta_{t}, \Delta_{C}$ or $\Delta_{C \cup i}$ are zero then part (a) of the Theorem is satisfied. If $\Delta_{i}, \Delta_{C}$ and $\Delta_{C u}$ are non-zero then the inequalities (4.1) and (4.2) must be strict. Putting $-b$, where $b>0$, for (4.2)'s left side, and formally solving it in combination with (4.3) for $\alpha_{i}$ gives

$$
\alpha_{i}=-b \Delta_{C} / \Delta_{C \cup i}
$$

This yields

$$
\Delta_{i} \Delta_{C} \Delta_{C U i}=-\left(J_{i i} \alpha_{i}\right)\left(\Delta_{C U i}\right)^{2} / b<0,
$$

by (4.1), which completes the proof of part (a) of Theorem 1 .

To prove (b), define the continuously differentiable functions

$$
f_{j}(\mathbf{x}, \mathbf{y}) \equiv J_{j}(\mathbf{x})-J_{j}(\boldsymbol{\sigma})-y_{j} \quad(j \in C \cup i)
$$

for any $\sigma$ satisfying $\Delta_{i} \Delta_{C} \Delta_{C \cup i}<0$, where $\mathbf{x}, \mathbf{y}$ are vectors with subscripts in $C \cup i$. Note that $\Delta_{C \cup i}$ must be nonzero. Clearly, $f_{j}(\sigma, 0) \equiv 0$ and the Jacobian of derivatives with respect to $\mathbf{x}$ is just $\Delta_{C u i} \neq 0$. By the implicit function theorem, then, there are unique continuously differentiable functions $\sigma_{j}+\delta_{j}$ $(j \in C \cup i)$ in the neighbourhood of $y=0$ such that

$$
J_{J}\left(\boldsymbol{\sigma}+\sum_{j \in C \cup i} \delta_{j} \mathbf{e}_{j}\right)=J_{j}(\boldsymbol{\sigma})+y_{j}
$$

Choosing $y_{t}=\beta, y_{j}=0(j \in C)$, where $\beta>0$ is sufficiently small, we have (2.3) and (2.4) and, since each $\delta_{j}$ is a continuously differentiable function of $\beta$, these equations hold for any $\delta_{i} \equiv \varepsilon$ sufficiently small and $\delta_{j}(j \in C) \rightarrow 0$ as $\varepsilon \rightarrow 0$.

Further, since $\Delta_{C \cup \imath} \neq 0$ we may find $\alpha,(j \in C \cup i)$, not all zero, satisfying (4.2) and (4.3) with strict inequality in (4.2). As above, we have (4.4) and deduce that

$$
J_{i i} \alpha_{i}=-b\left(\Delta_{i} \Delta_{C} \Delta_{C \cup i}\right) /\left(\Delta_{C \cup i}\right)^{2}>0 .
$$

But remember that (4.2) and (4.3) also come from linearization of equations like (2.3) and (2.4) so we may identify these $\alpha$ 's with those from such a procedure. 
This shows that $\varepsilon$ and $\alpha_{i}$ have the same sign, whence

$$
J_{u i} \varepsilon>0 \text {. }
$$

Assume that (2.2) is not true for all $\varepsilon$ sufficiently small $j$; then there must be a sequence $\left\{\varepsilon^{\prime}\right\}$ of $\varepsilon$ values tending monotonically to zero such that

$$
J_{i}\left(\boldsymbol{\sigma}+\varepsilon^{\prime} \mathbf{e}_{i}\right) \leqslant J_{j}(\boldsymbol{\sigma}) \text {, }
$$

and this contradicts (4.9). Thus, if $\Delta_{i} \Delta_{C} \Delta_{C u i}<0$, then, for any $\varepsilon$ sufficiently small, we have suitable $\delta_{j}(j \in C)$ such that (2.2), (2.3) and (2.4) hold; that is, $\boldsymbol{\sigma} \in D_{i, c}$.

The 'only if' part of Theorem 2 is effectively proved in the course of the proof of Theorem 1(b); the uniqueness of the $\alpha_{j}, j \in C$, follows from $\Delta_{C} \neq 0$. Conversely, assume (2.8), (2.9) and (2.10) hold and let $-b$ be the right side of (2.9). If we suppose $\Delta_{C \cup i}=0$, then the vectors $\left\{J_{k j}, j \in C \cup i\right\}$ for $k \in C \cup i$ are linearly dependent, that is, there exists $\mu_{k}(k \in C \cup i)$, not all zero, such that

$$
\sum_{k \in C \cup \imath} \mu_{k} J_{k j}=0 \quad(j \in C \cup i)
$$

If $\mu_{i}=0$, then (4.11) implies that $\Delta_{C}=0$ and hence, for given $\alpha_{i}$, the solution of (2.9) and (2.10) for $\alpha_{j}(j \in C)$ and $b$ cannot be unique. Thus $\mu_{i} \neq 0$ and, if $\lambda_{k}=-\left(\mu_{k} / \mu_{i}\right)(k \in C),(4.7)$ becomes

$$
J_{i j}=\sum_{k \in C} \lambda_{k} J_{k j}
$$

This implies that

$$
\sum_{j \in C \cup i} J_{i j} \alpha_{j}=\sum_{k \in C} \lambda_{k} \sum_{j \in C \cup i} J_{k j} \alpha_{j}
$$

which contradicts (2.9) and (2.10). Thus $\Delta_{C u i} \neq 0$. Now (4.4) holds, implying (4.5) and completing the proof of Theorem 2.

\section{Proof of Theorem 4}

We recall that a Cournot optimum $\sigma$ satisfies the condition that

$$
J_{i}\left(\boldsymbol{\sigma}+\varepsilon \mathbf{e}_{i}\right)<J_{i}(\boldsymbol{\sigma})
$$

for every sufficiently small $\varepsilon \neq 0$, and all $i$. From the part (2.1) of the definition of $D_{i, C}$ it follows that $\sigma \in D_{i, C}$ for all $i$ and $C$, and hence that $\sigma \in O_{\mathrm{IV}}$.

Now choose $\sigma \in P O^{\prime}$. If

$$
J_{1}\left(\boldsymbol{\sigma}+\varepsilon \mathbf{e}_{1}\right)<J_{1}(\boldsymbol{\sigma})
$$

for any sufficiently small $\varepsilon \neq 0$, then $\sigma \in D_{1, \Lambda-1}$ by (2.1). Otherwise, we can 
choose an arbitrarily small $\varepsilon \neq 0$ such that

$$
J_{1}\left(\boldsymbol{\sigma}+\varepsilon \mathbf{e}_{1}\right)>J_{1}(\boldsymbol{\sigma}),
$$

because, by assumption, $J_{1}$ is not constant on any interval of values of $\sigma_{1}$. By the definition of $P O^{\prime}, \Delta_{\Lambda-1} \neq 0$. By the implicit function theorem, we can therefore find $\delta_{2}, \ldots, \delta_{N}$ such that

$$
J_{i}\left(\sigma_{1}+\varepsilon, \sigma_{2}+\delta_{2}, \ldots, \sigma_{N}+\delta_{N}\right)=J_{i}(\sigma), \quad i=2, \ldots, N .
$$

But $\sigma$ is Pareto optimal, so that

$$
J_{i}\left(\sigma_{1}+\varepsilon, \sigma_{2}+\delta_{2}, \ldots, \sigma_{N}+\delta_{N}\right)<J_{i}(\sigma)
$$

for at least one $i$, which can only be $i=1$. Thus (2.2), (2.3) and (2.4) are satisfied if $C$ is $\Lambda-1$, so that again $\sigma \in D_{1, \Lambda-1}$. Similarly, $\sigma \in D_{i, \Lambda-i}$ for all $i$, and Theorem 4 is proved.

\section{Proofs of Theorems 5 and 6}

If $\sigma \in O_{1}^{W}$, then $\sigma \in D_{\imath, C}^{W}$ so that

$$
\Delta_{i} \Delta_{C} \Delta_{C u i}<0
$$

for at least one $i$ and $C$. If $\Delta_{i}=0$, we choose

$$
A_{j k}= \begin{cases}1 & \text { if } j=k=i \\ 0 & \text { if not }\end{cases}
$$

which is psd and satisfies (1.1). If $\Delta_{C}=0$, we choose

$$
A_{j k}= \begin{cases}x_{j} x_{k} & \text { for } j, k \in C, \\ 0 & \text { if not, }\end{cases}
$$

where the $x_{j}$ are a non-zero solution of the equations

$$
\sum_{k \in C} x_{k} J_{J k}=0 \quad \text { for all } j \in C .
$$

Again $A_{j k}$ is psd and satisfies (1.1). The case where $\Delta_{C u t}=0$ is treated similarly. If any combination of $\Delta_{l}=0, \Delta_{C}=0$ and $\Delta_{C \cup i}=0$ holds, one has a choice of the corresponding $A_{j k}$ 's.

The remaining case is $\Delta_{i} \Delta_{C} \Delta_{C \cup i}<0$, which, by Theorem 2 , is equivalent to the relations (2.8), (2.9) and (2.10). From (2.8) and (2.9) there is a $q>0$ such that

$$
\sum_{j \in C \cup i} J_{i j} \alpha_{j}\left(1+q \delta_{i j}\right)=0
$$

where $\delta_{i j}$ is the Krönecker delta. Multiplying (6.5) by $\alpha_{i}$, and the $k$ th equation of (2.10) by $\alpha_{k}$, for all $k \in C$, gives

$$
\sum_{j \in \Lambda} J_{k j} A_{k j}=0
$$


where

$$
A_{k j}= \begin{cases}\alpha_{i}^{2}(1+q) & \text { if } k=j=i \\ 0 & \text { if } k \notin C \cup i \text { or } j \notin C \cup i \text { or both, } \\ \alpha_{k} \alpha_{j} & \text { otherwise, }\end{cases}
$$

which is symmetric and psd. Thus Theorem 5 is proved.

Although Theorem 6 was proved in Section 3, it is perhaps useful to give a simple alternative proof which does not use the determinantal relation (3.9) or the results of [3]. From Theorem 5 we need prove only that the matrix condition (1.1) implies the optimum $O_{1}^{W}$. For $N=2$ and $A_{12} \neq 0,(1.1)$ can be written

$$
\begin{aligned}
& J_{11} \alpha_{1}+J_{12} \alpha_{2}=-\left(J_{11} \alpha_{1}\right) a, \\
& J_{21} \alpha_{1}+J_{22} \alpha_{2}=0
\end{aligned}
$$

where

$$
a=\left(A_{11} A_{22}-A_{12} A_{21}\right) / A_{12}^{2}
$$

and

$$
\alpha_{2}=\alpha_{1} A_{22} / A_{12}
$$

with any $\alpha_{1} \neq 0$. Now choose $\alpha_{1}$ such that $J_{11} \alpha_{1}>0$, so that (6.8) gives $J_{11} \alpha_{1}+J_{12} \alpha_{2}<0$ if $\operatorname{det}\left(A_{i j}\right)>0$. Thus (2.8), (2.9) and (2.10) hold in this case, so that $\sigma \in D_{1,2}{ }^{S} \subseteq D_{1,2}{ }^{W}$. If $\operatorname{det}\left(A_{i j}\right)=0$, then $a=0$, so that (6.8) and (6.9) imply that $\Delta_{12}=0$ and so $\sigma \in D_{1,2}{ }^{W}$ again.

In the case $A_{12}=0,(1.1)$ reduces to $\Delta_{1}=0$ or $\Delta_{2}=0$ or both, so again $\sigma \in D_{1,2}{ }^{W}$.

Similarly one proves that $\sigma \in D_{2,1}{ }^{W}$ and equivalently $\sigma \in O_{1}^{W}$, which proves Theorem 6.

\section{Proof of Theorem 7}

Suppose, contrary to the assumption of Theorem 7 , that the $\Delta^{(i)}$ are all positive. Then the symmetric matrix $H_{i} J_{i j}+H_{j} J_{j i}$ is positive definite, and consequently

$$
\sum_{i, j} x_{i}\left(H_{i} J_{i j}+H_{j} J_{j i}\right) x_{j}>0
$$

for any $x_{1}, \ldots, x_{N}$ not all zero. Equivalently

$$
\sum_{i, j} x_{i} H_{i} J_{i j} x_{j}>0
$$


We write $A_{i j}=\Sigma_{k} B_{i k} B_{j k}$ for the $A_{i j}$ in (1.1), where $B_{i j}$ is a non-zero matrix. Thus

$$
\sum_{i, j} B_{i k} H_{i} J_{i j} B_{j k} \geqslant 0 \text { for all } k
$$

with the strict $>$ holding for at least one $k$. Then

$$
\begin{aligned}
0 & <\sum_{i, j, k} B_{i k} H_{i} J_{i j} B_{j k} \\
& =\sum_{i} H_{i} \sum_{j} J_{i j} \sum_{k} B_{i k} B_{j k} \\
& =\sum_{i} H_{i} \sum_{j} J_{i j} A_{i j} .
\end{aligned}
$$

But the right side is zero by the condition (1.1), which is a contradiction. Thus the $\Delta^{(i)}$ are not all positive. Similarly they do not satisfy (3.10) since this would imply that $H_{i} J_{i j}+H_{j} J_{j i}$ be negative-definite and lead again to a contradiction. This completes the proof of Theorem 7.

\section{Proof of Theorem 8}

Let $P$ be the (non-empty) set of $i$ values for which $A_{i t}>0$, and now restrict suffices to $P$. First suppose that $J_{i j}<0$ for all $i \neq j$ and that the $2 \times 2$ principal minor determinants (pmd) of $A_{i j}$, are non-zero. Then (1.1) implies that

$$
-L_{i} \equiv \sum_{j \in P} J_{i j} A_{i i}{ }^{1 / 2} A_{i j}{ }^{1 / 2}=\sum_{j \in P} J_{i j}\left(A_{u}{ }^{1 / 2} A_{i j}{ }^{1 / 2}-A_{i j}\right)<0
$$

using the fact that $A_{i j}=0$ for $j \notin P$. Since

$$
\sum_{j \in P}\left(J_{i j} A_{i i}{ }^{1 / 2} A_{j j}{ }^{1 / 2}+L, \delta_{i j}\right) \equiv 0
$$

it follows that

$$
0=\operatorname{det}\left(J_{i j} A_{i i}{ }^{1 / 2} A_{j j}{ }^{1 / 2}+L_{j} \delta_{i j}\right)=\sum_{s \subseteq P} \Gamma_{S} \Delta_{s} L_{P-S}
$$

where

$$
\begin{aligned}
& L_{C} \equiv \prod_{i \in C} L_{i}>0, \\
& \Gamma_{C} \equiv \prod_{i \in C} A_{i i}>0
\end{aligned}
$$

for any $C \subseteq P$. Here $\Delta_{\varnothing}=1$, where $\varnothing$ is the empty set.

Clearly, the region $B_{0}$ is excluded in this case. 
If only a subset of the $2 \times 2$ pmd's are non-zero, then (8.3) still holds but $L_{C}>0$ only for $C \subseteq T \subset P$, say. Thus (8.3) effectively becomes

$$
0=\sum_{S \supseteq P-T} \Gamma_{S} \Delta_{S} L_{P-S}
$$

which leads to an excluded region that contains $B_{0}$. Since the inadmissible $\sigma$ 's are those which fall in an excluded region for all psd $A_{i j}$ 's, the theorem is proved for non-zero $J_{i j}(i \neq j)$.

If some $J_{i,}=0(i \neq j)$ some further $L_{i}$ may be zero and, as above, this merely generates excluded regions containing $B_{0}$. In the extreme case, for example, where $L_{i}=0$ for all $i,(8.3)$ reduces to $\Gamma_{P} \Delta_{P}=0$, which implies $\Delta_{P}=0$ and excludes $B_{0}$ once again.

Proving part (b) of Theorem 8, we note the assumption $J_{i j}<0$ for all $i \neq j$. From each of the conditions in (1.1) excluding the $i$ th we have

$$
A_{i j}=A_{j i}=-\sum_{k \in \Lambda-i}\left(A_{j k} J_{j k} / J_{j i}\right)
$$

for $j \neq i$. Substituting these in the $i$ th equation gives

$$
J_{i i} A_{u}=\sum_{j \in \Lambda-i} \frac{J_{i j}}{J_{j k}} \sum_{k \in \Lambda-i} J_{j k} A_{j k} \geqslant \sum_{j \in \Lambda-i} \frac{J_{i j}}{J_{j i}} \sum_{k \in \Lambda-i-j} J_{j k} A_{j k},
$$

where we have used the condition $J_{j j}>0$ for all $j \neq i$, involved in $B_{i}$. Since $A$ is psd we have $A_{j k} \leqslant A_{j j}{ }^{1 / 2} A_{k k}{ }^{1 / 2}$. With the negativity of $J_{i j}$ we have

$$
\begin{aligned}
J_{i i} A_{i i} & \geqslant \sum_{, \in \Lambda-i} \frac{J_{i j}}{J_{j i}} A_{j j}{ }^{1 / 2} \sum_{k \in \Lambda-t-j} J_{j k} A_{k k}{ }^{1 / 2} \\
& =\sum_{(j, k) \subseteq \Omega_{i}}\left[\frac{1}{N-2}\left(\dot{A_{j j}} \frac{J_{i j}}{J_{j i}} J_{j j}+A_{k k} \frac{J_{t k}}{J_{k t}} J_{k k}\right)+A_{j j}{ }^{1 / 2} A_{k k}{ }^{1 / 2}\left(\frac{J_{i j}}{J_{j t}} J_{j k}+\frac{J_{t k}}{J_{k t}} J_{k j}\right)\right],
\end{aligned}
$$

where $\Omega_{i}$ is the set $\{j \in \Lambda-i, k \in \Lambda-i, j \neq k\}$. Again using the condition $J_{j j}>0$ for $j \in \Lambda-i$ and applying the inequality $x+y>2 x^{1 / 2} y^{1 / 2}$ for $x, y>$ 0 to the term in the first round brackets gives

$$
J_{i i} A_{i i} \geqslant \sum_{(j, k) \subseteq \Omega_{i}} A_{j j}{ }^{1 / 2} A_{k k}{ }^{1 / 2}\left(\frac{J_{i j} J_{i k}}{J_{j i} J_{k l}}\right)^{1 / 2}\left[\frac{2}{N-2}\left(J_{j j} J_{k k}\right)^{1 / 2}-J_{j k} X-J_{k j} X^{-1}\right] \text {, }
$$

with $X$ given by (3.12).

Various cases need to be considered. If $A_{i i}>0$ then $B_{i}$ is immediately excluded. If $A_{i i}=0$ then $A_{j j}>0$ for at least one $j \neq i$ : if there is only one such $j$ then (1.1) reduces to $J_{j j}=0$, which excludes $B_{i}$; if both $A_{j j}>0$ and $A_{k k}>0$ then $B_{i}$ is excluded by the condition $J_{j j} J_{k k}>E_{i j k}$. 


\section{Acknowledgement}

We are indebted to a referee for a particularly careful reading of the manuscript and for suggesting that the implicit function theorem be used to improve the proofs of Theorems 1 and 4 .

\section{References}

[1] R. H. Day, "Adaptive processes and economic theory", in Adaptive economic models (eds. R. H. Day and T. Groves), (Academic Press, New York, 1975), 1-38.

[2] D. J. Gates and J. A. Rickard, "Pareto optimum by independent trials", Bull. Austral. Math. Soc. 12 (1975), 259-265.

[3] D. J. Gates, J. A. Rickard and D. J. Wilson, “A convergent adjustment process for firms in competition", Econometrica, 45 (1977), 1349-1364.

[4] D. J. Gates, J. A. Rickard and D. J. Wilson, "Convergence of a market related game strategy", J. Math. Economics, 5 (1978), 97-109.

[5] D. J. Gates, J. A. Rickard and M. Westcott, "Exact cooperative solutions of a duopoly model without cooperation", J. Math. Economics (to appear).

[6] D. J. Gates and M. Westcott, "Extended optima and equilibria for continuous games. II. A class of economic models", preprint, second paper of this series (1979).

[7] D. J. Gates and M. Westcott, "Extended optima and equilibria for continuous games. III. Comparison with experiment", preprint, third paper of this series (1979).

[8] A. P. Kirman, "Learning by firms about demand conditions", in R. H. Day and T. Groves, loc. cit. 137-156.

[9] J. P. Mayberry, J. F. Nash and M. Shubik, "A comparison of treatments of a duopoly situation", Econometrica, 21 (1953), 141-154.

[10] J. von Neumann and O. Morgenstern, Theory of games and economic behaviour, 2 nd ed. (Princeton University Press, 1947).

[11] D. J. Seidmann, "The rationality of expections equilibria", preprint, London School of Economics, 1979.

C. S. I. R. O. Division of Mathematics and Statistics

P. O. Box 1965

Canberra, ACT 2601 